\title{
Magnetic Self-Assembly for the Synthesis of Magnetically Exchange Coupled $\mathrm{MnBi} / \mathrm{Fe}$-Co Composites
}

\author{
Xia Xu ${ }^{a}$, Yang-Ki Hong ${ }^{b^{*}}$, Jihoon Park $^{b}$, Woncheol Lee $^{\mathrm{b}}$, Alan M. Lane ${ }^{\mathrm{a}}$, and Jun Cui ${ }^{\mathrm{c}}$ \\ ${ }^{a}$ Department of Chemical and Biological Engineering and MINT Center, The University of \\ Alabama, Tuscaloosa, AL 35487 USA \\ ${ }^{\mathrm{b}}$ Department of Electrical and Computer Engineering and MINT Center, The University of \\ Alabama, Tuscaloosa, AL 35487 USA \\ ${ }^{\mathrm{c}}$ Energy and Environment Directorate, Pacific Northwestern National Laboratory, Richland, WA \\ 99354, USA
}

\begin{abstract}
:
Exchange coupled hard/soft $\mathrm{MnBi} / \mathrm{Fe}-\mathrm{Co}$ core/shell structured composites were synthesized using a magnetic self-assembly process. MnBi particles were prepared by arc-melting, and Fe-Co nanoparticles were synthesized by an oleic acid assisted chemical reduction method. Grinding a mixture of micron-sized $\mathrm{MnBi}$ and $\mathrm{Fe}-\mathrm{Co}$ nanoparticles in hexane resulted in $\mathrm{MnBi} / \mathrm{Fe}-\mathrm{Co}$ core/shell structured composites. The MnBi/Fe-Co (95/5 wt.\%) composites showed smooth magnetic hysteresis loops, enhanced remanent magnetization, and positive values in the $\Delta M$ curve, indicating exchange coupling between $\mathrm{MnBi}$ and $\mathrm{Fe}-\mathrm{Co}$ particles.
\end{abstract}

Keywords: MnBi, FeCo, exchange coupling, core/shell, rare-earth-free.

"Corresponding author: phone: 205-348-7268; fax: 205-348-6959; email: ykhong@eng.ua.edu 


\section{Introduction}

For past half century, rare-earth (RE) ferromagnetic materials (Nd-Fe-B and Sm-Co) have dominated permanent magnets for energy applications, including electric motors, wind turbines, and other electric machines [1-3]. The figure of merit of permanent magnets is the maximum energy product $\left[(B H)_{\max }\right]$. The $(B H)_{\max }$ can be estimated as $(B H)_{\max }=\left(B_{\mathrm{r}}\right)^{2} / 4$ for $H_{\mathrm{ci}}>B_{\mathrm{r}} / 2$ or $(B H)_{\max }=\left(B_{\mathrm{r}}-H_{\mathrm{ci}}\right) H_{\mathrm{ci}}$ for $H_{\mathrm{ci}}<B_{\mathrm{r}} / 2$, where $B_{\mathrm{r}}$ is the remanent magnetization, and $H_{\mathrm{ci}}$ is the coercivity of magnetic materials. Therefore, both high magnetization and coercivity are required for a high $(B H)_{\max }$. The Nd-Fe-B magnet shows the highest $(B H)_{\max }$ among all permanent magnets at room temperature, with a world record value of 59 MGOe [4].

However, there are two issues to be addressed for continued use of RE permanent magnets: low Curie temperature and limited supply of RE metals. The Curie temperature of Nd-Fe-B is $580 \mathrm{~K}$ [4], which limits its application in electrical motors. Also, the supplies and cost of RE metals are not stable in international markets. Therefore, much effort has been made in the development of RE free permanent magnets with high $(B H)_{\max }$ [5-9]. One of the promising candidates for RE free permanent magnets is the low temperature phase (LTP) phase $\mathrm{MnBi}$, with a theoretical $(B H)_{\max }$ of $17 \mathrm{MGOe}$, coercivity of $17 \mathrm{kOe}$ at room temperature [10], and Curie temperature of $628 \mathrm{~K}$ [11]. Furthermore, the anisotropy constant of LTP MnBi increases as temperature increases up to $523 \mathrm{~K}$ because of its positive temperature coefficient $(\Delta K / \Delta T>0)[12,13]$. However, one of the drawbacks of $\mathrm{MnBi}$ is its relatively low magnetization, with $\sim 60 \mathrm{emu} / \mathrm{g}$ at room temperature [14]. To solve this problem, Hong et al. investigated the effects of interstitial Co and Fe-Co doping on LTP MnBi using first principle calculations [15]. After doping, the results show increased anisotropy constant and magnetization, but decreased Curie temperature.

Besides doping, the principle of magnetic exchange coupling provides another solution to improve the magnetization and $(B H)_{\max }$ of a single hard phase magnet [16-18]. The magnetically exchange coupled magnet is composed of both soft and hard phases, and makes use of the high magnetization of the soft phase and high coercivity of the hard phase. Effective exchange coupling requires the size of the soft phase to be no more than twice the domain wall thickness of the hard phase, usually less than $30 \mathrm{~nm}$. A magnetically exchange coupled LTP MnBi/soft phase (Fe or Co) composite was synthesized previously 
by either a physical [14] or a chemical [19] method. However, in the physical method, the agglomeration of soft phase particles results in Co size larger than $10 \mu \mathrm{m}$, resulting in weak exchange coupling between hard and soft phases. In the chemical method, the as-synthesized MnBi/Co core/shell particles show low coercivity $(<2000 \mathrm{Oe})$, which results in a low $(B H)_{\max }$.

In this paper, we report a magnetic self-assembly method (physical method) to synthesize $\mathrm{MnBi} / \mathrm{Fe}$ Co composites. The effective magnetic exchange coupling between MnBi and $\mathrm{Fe}-\mathrm{Co}$ is well demonstrated by homogenous distribution between hard and soft phases, a smooth magnetic hysteresis loop, and a positive peak in the $\Delta M$ curve.

\section{Experimental}

$\mathrm{MnBi}$ and $\mathrm{Fe}-\mathrm{Co}$ particles were separately synthesized before the synthesis of $\mathrm{MnBi} / \mathrm{Fe}-\mathrm{Co}$ composites. The MnBi raw particles were synthesized by arc melting, followed by grinding, annealing and ball milling [8]. Fe-Co nanoparticles were synthesized using oleic acid assisted reduction based on a previous report [20] by replacing $\mathrm{MnCl}_{2}$ with $\mathrm{FeCl}_{2}$ and $\mathrm{CoCl}_{2}$, and the molar ratio of $\mathrm{Fe}$ to Co was controlled at 65/35.

To synthesize MnBi/Fe-Co composites, a mixture of MnBi raw particles and Fe-Co nanoparticles was ground for $5 \mathrm{~min}$ in $20 \mathrm{~mL}$ hexane in a glove box. The total mass of $\mathrm{MnBi}$ and $\mathrm{Fe}-\mathrm{Co}$ particles was controlled to $0.1 \mathrm{~g}$, and the mass of Fe-Co nanoparticles was controlled to $0.005 \mathrm{~g}$ and $0.01 \mathrm{~g}$ in two parallel experiments, and the corresponding products were identified as $\mathrm{MnBi} / \mathrm{Fe}-\mathrm{Co}(95 / 5 \mathrm{wt} \%)$ and $\mathrm{MnBi} / \mathrm{Fe}-\mathrm{Co}$ (90/10 wt.\%), respectively. As a comparison, pure MnBi raw particles were also ground under the same conditions.

The particles and composites were characterized with a vibrating sample magnetometer (VSM,

Microsense 3473-70), X-ray diffractometer (XRD, Bruker D8, Cu-Ka radiation $(\lambda=1.541 \AA$ )), transmission electron microscope (TEM, FEI TECNAI F-20) and scanning electron microscope (JEOL 7000) for magnetic properties, crystal phases, particle morphology and elemental mappings, respectively. Sample preparation for VSM and XRD characterization was conducted in an Ar atmosphere glove box to prevent oxidation. 


\section{Results and discussion}

Figure 1 shows SEM image and particle size distribution of MnBi raw and ground particles. The size of $\mathrm{MnBi}$ raw particles is in the range from 5 to $55 \mu \mathrm{m}$, and follows a double Gaussian distribution, with an average size of $9.3 \mu \mathrm{m}$ and $38.5 \mu \mathrm{m}$, respectively, as shown in Fig. 1a and c. After 5 min grinding, the size range is narrowed and the average size is decreased to $5.2 \mu \mathrm{m}$. The corresponding SEM image and particle size distribution for 5 min ground MnBi particles are shown in Fig. $1 \mathrm{~b}$ and d.
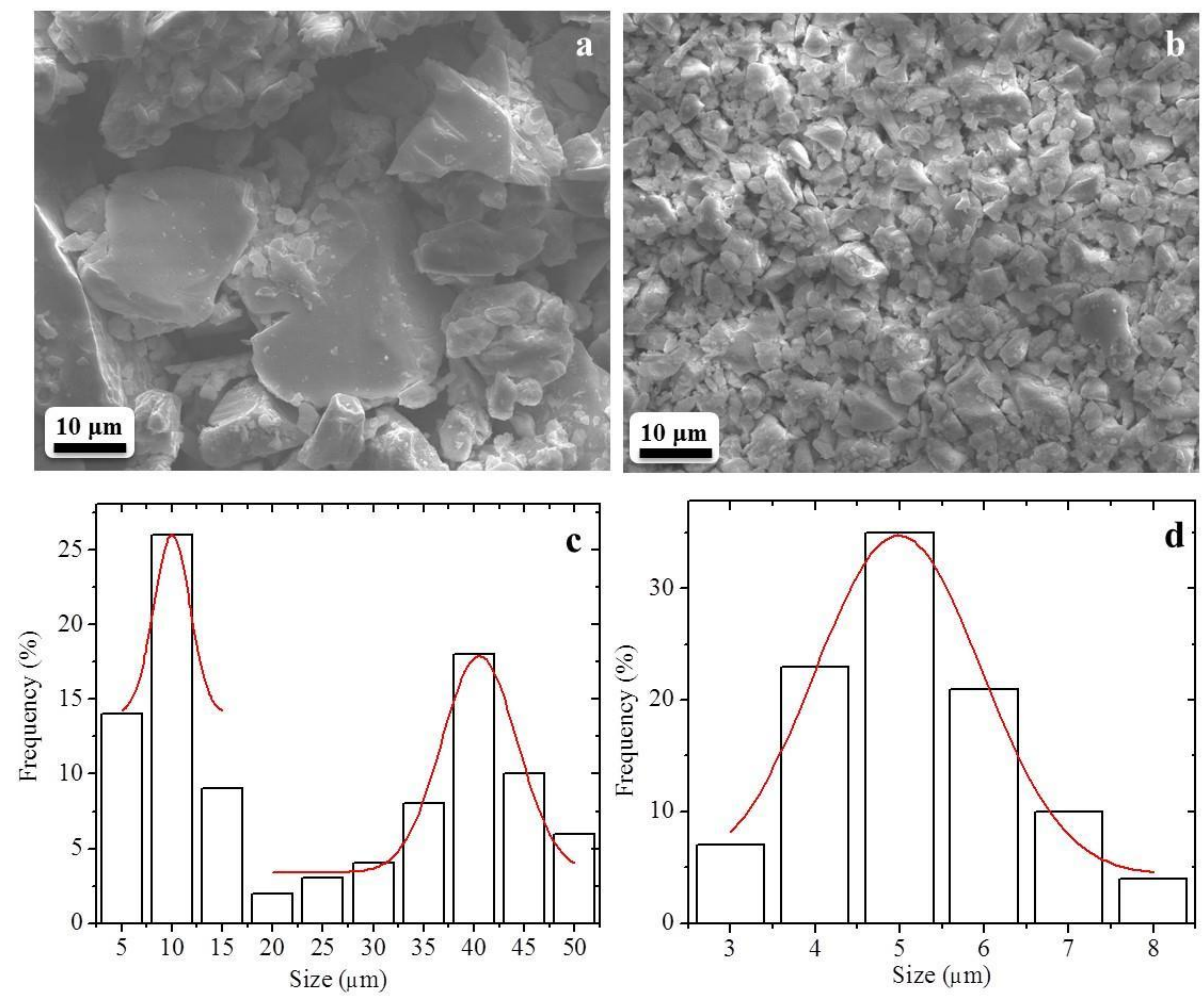

Fig. 1. SEM images of MnBi particles and their size distribution: (a) and (c) SEM image of MnBi raw particles and their size distribution; (b) and (d) SEM image of ground MnBi particles and their size distribution.

The magnetic hysteresis loops of raw and 5 min ground MnBi particles are shown in Fig. 2. After grinding, there is negligible change in the saturation magnetization $(M)$, while the coercivity $\left(H_{c}\right)$ increases from $2 \mathrm{kOe}$ to $8.2 \mathrm{kOe}$. The increased $H_{c}$ can be attributed to the decrease in particle size. The inset of Fig. 2 shows a general relationship between particle size and $H_{c}$ in magnetic materials [21]. The highest $H_{\mathrm{c}}$ appears at the single domain size $\left(\mathrm{d}_{0}\right)$. In the case of $\mathrm{MnBi}$, the single domain size is around 
$0.5 \mu \mathrm{m}$, and the corresponding $H_{c}$ is about $17 \mathrm{kOe}$ [22]. Grinding reduces the size of MnBi particles from $\mathrm{d}_{1}$ to $\mathrm{d}_{2}$, thereby, a dramatic increase in $H_{c}$. The XRD patterns of MnBi before and after grinding in Fig. 3 show a negligible change in the crystal structure.

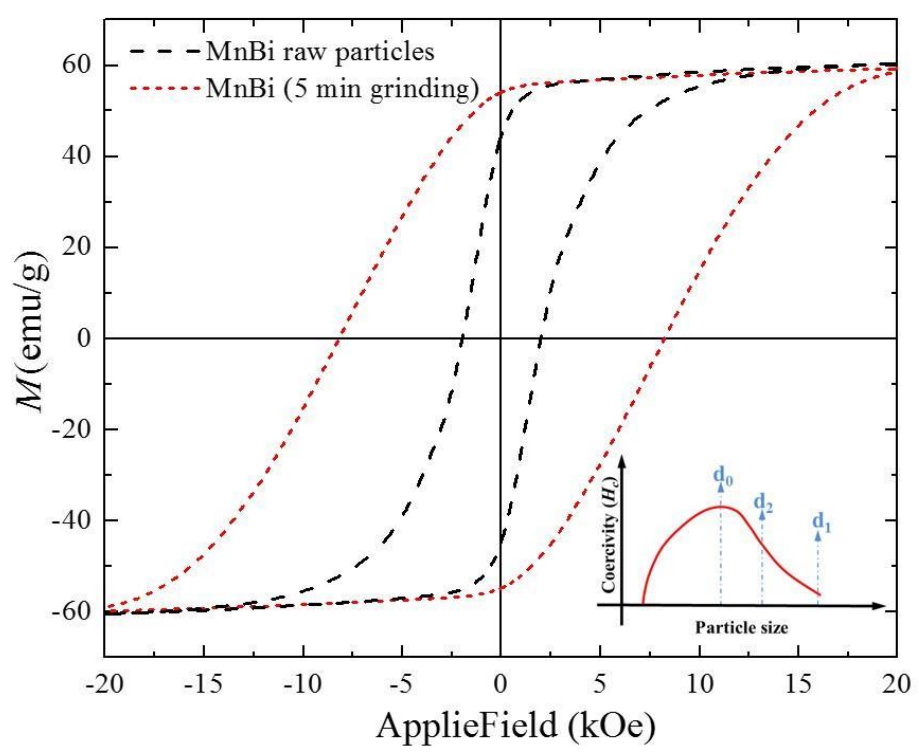

Fig. 2. Magnetic hysteresis loops of MnBi raw particles and ground particles for $5 \mathrm{~min}$.

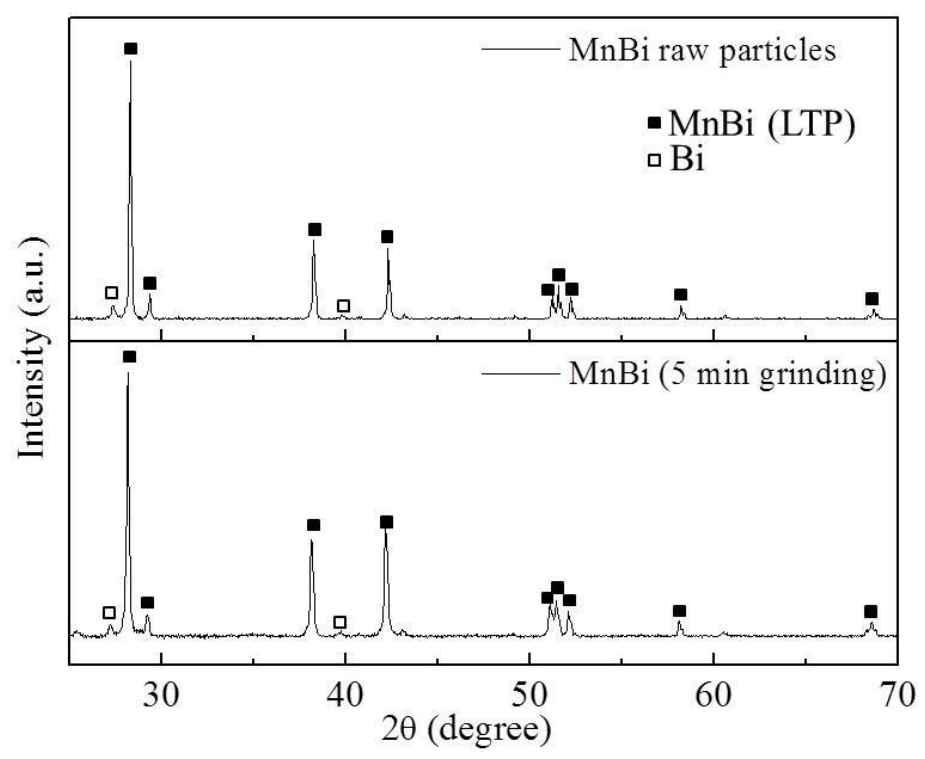

Fig. 3. XRD patterns of MnBi raw particles and ground particles for 5 min.

Figure 4 shows a magnetic hysteresis loop, TEM image and XRD patterns of Fe-Co nanoparticles. The $M$ and $H_{c}$ are $151 \mathrm{emu} / \mathrm{g}$ and 41 Oe, respectively. Fe-Co particles are mono-dispersed [20], and the size is between 5 to $10 \mathrm{~nm}$. The presence of a complex of cyclodextrin with oleic acid adhered to Fe-Co 
nanoparticle surface leads to well dispersed Fe-Co nanoparticles in hexane [23]. XRD patterns confirm the body centered cubic (BCC) Fe-Co phase, as shown in the bottom inset of Fig. 4. The crystallite size of Fe-Co particles is calculated using Scherrer equation [24]:

$$
\tau=\frac{K \lambda}{\beta \cos \theta},
$$

where $\tau$ is the mean crystallite size, $K$ is the dimensionless shape factor $(K=0.9), \lambda$ is the $X$ ray wavelength $(\lambda=0.154 \mathrm{~nm}), \beta$ is the line broadening at half the maximum intensity $(\beta=0.007$, in radians), and $\theta$ is the Bragg angle $\left(\theta=22.4^{\circ}\right)$. We found that the calculated crystallite size is $21 \mathrm{~nm}$.

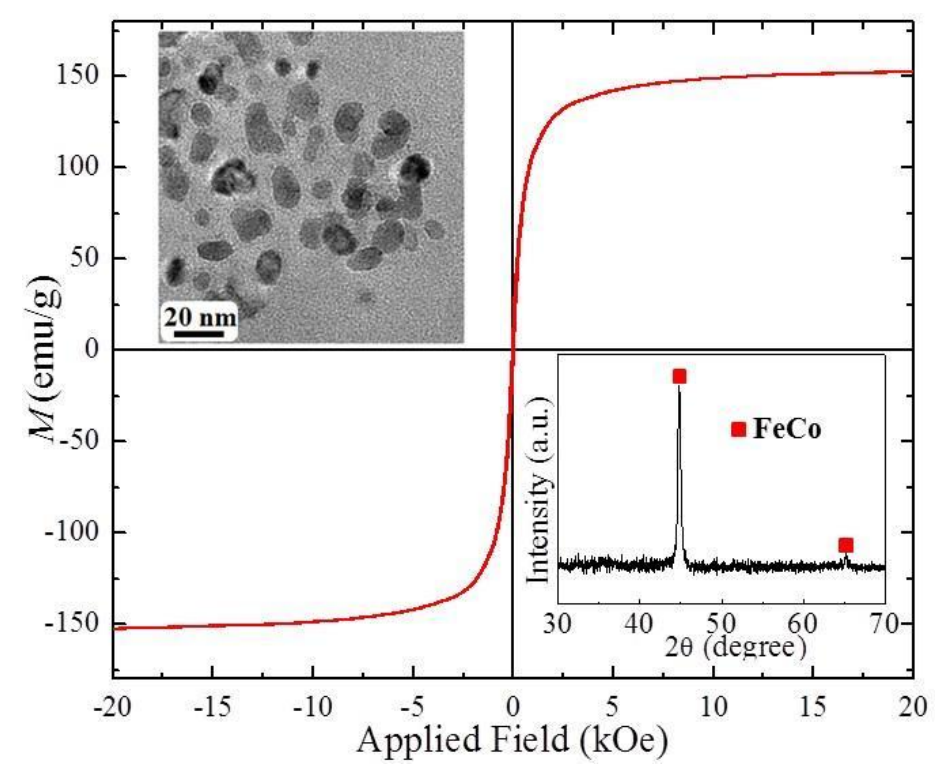

Fig. 4. Magnetic hysteresis loop, TEM image and XRD pattern of the Fe-Co nanoparticles.

Figure 5 shows magnetic hysteresis loops of MnBi particles and MnBi/Fe-Co (95/5 wt.\% and 90/10 wt.\%) composites after 5 min grinding. The particles and composites were aligned in a magnetic field of $16 \mathrm{kOe}$ before the hysteresis loop measurement. MnBi particles show $60 \mathrm{emu} / \mathrm{g} M$ and $8.2 \mathrm{kOe} H_{\mathrm{c}}$. With increasing the mass of Fe-Co particles, the $M$ of the $\mathrm{MnBi} / \mathrm{Fe}-\mathrm{Co}$ composite increases, and $H_{c}$ decreases. $\mathrm{MnBi} / \mathrm{Fe}-\mathrm{Co}(95 / 5$ wt.\%) composites show a smooth magnetic hysteresis loop, and increased remanent magnetization $\left(M_{r}\right)$ from MnBi particles, indicating single-phase-like magnetic behaviors and exchange coupling between hard and soft phases. However, MnBi/Fe-Co (90/10 wt.\%) composites show a decreased $M_{r}$ from MnBi particles, indicating a weak exchange coupling [25]. 


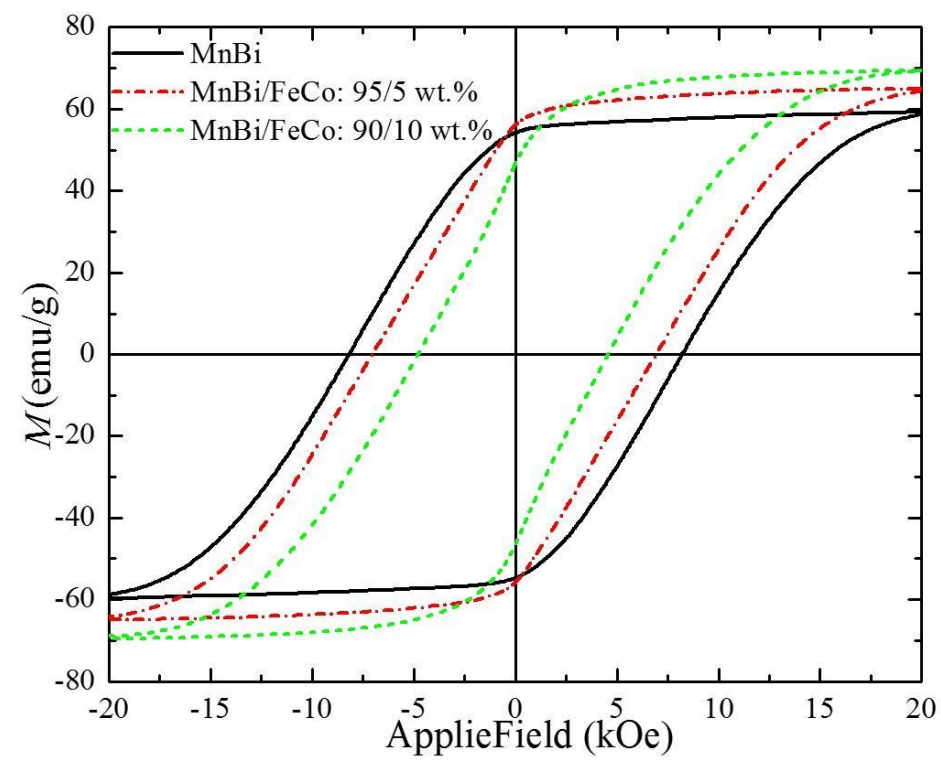

Fig. 5. Magnetic hysteresis loops of MnBi particles and MnBi/Fe-Co (95/5 wt.\% and 90/10 wt.\%) composites after 5 min grinding.

To further understand the exchange coupling between MnBi and Fe-Co particles, Henkel plots of $\mathrm{MnBi}$ and $\mathrm{MnBi} / \mathrm{Fe}-\mathrm{Co}$ particles are shown in Fig. 6. The Henkel plot has been applied to describe the magnetic interactions among particles, and it is defined as $\Delta M=M_{\mathrm{d}}(H)-\left[1-2 M_{\mathrm{r}}(H)\right][14,15]$, where $M_{\mathrm{d}}$ is the normalized demagnetization remanence, $M_{\mathrm{r}}$ is the normalized isothermal magnetization remanence, and $H$ is the applied magnetic field. Both positive and negative $\Delta M$ values are observed in $\mathrm{MnBi} / \mathrm{Fe}-\mathrm{Co}$ composites, but only negative $\Delta M$ values in MnBi particles. Positive $\Delta M$ values indicate exchange coupling between magnetically hard $\mathrm{MnBi}$ and soft Fe-Co phases [26]. Negative values indicate magnetostatic interaction among particles [26]. Therefore, exchange coupling dominates in $\mathrm{MnBi} / \mathrm{FeCo}$ (95/5 wt.\%) composites, and magnetostatic interaction dominates in $\mathrm{MnBi} / \mathrm{Fe}-\mathrm{Co}$ (90/10 wt.\%) composites. This could be attributed to the larger amount of Fe-Co nanoparticles in $\mathrm{MnBi} / \mathrm{Fe}-\mathrm{Co}(90 / 10$ wt.\%) composites, and that not all the Fe-Co nanoparticles are well exchange coupled with MnBi, which results in less effective exchange coupling and decreased $M_{r}$ in Fig. 5. 


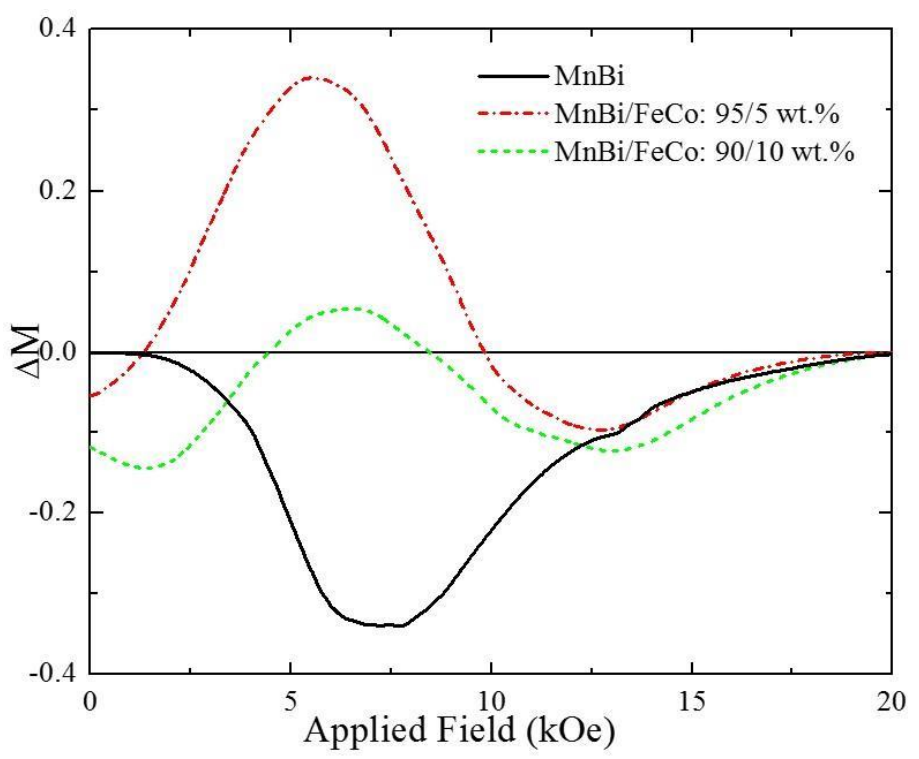

Fig. 6. $\Delta M$ curves of $\mathrm{MnBi}$ particles and $\mathrm{MnBi} / \mathrm{Fe}-\mathrm{Co}(95 / 5 \mathrm{wt} . \%$ and 90/10 wt.\%) composites.

XRD patterns of $\mathrm{MnBi}$ particles and $\mathrm{MnBi} / \mathrm{Fe}-\mathrm{Co}$ (95/5 wt.\% and 90/10 wt.\%) composites are presented in Fig. 7. The Fe-Co peak appears in both MnBi/Fe-Co (95/5 wt.\% and 90/10 wt.\%) composites, and higher mass percentage results in a stronger Fe-Co peak intensity.

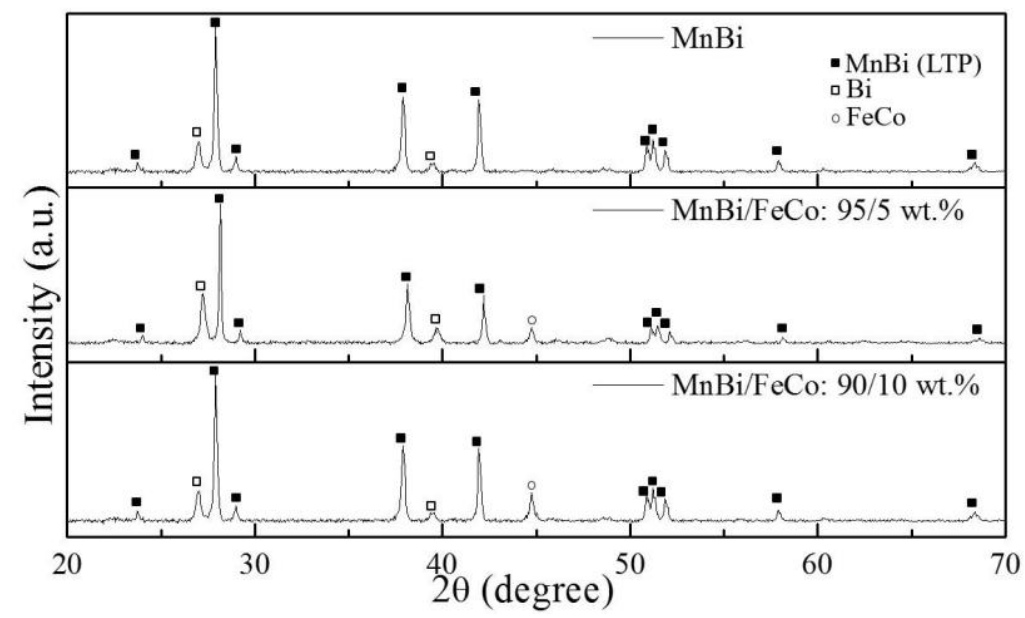

Fig. 7. Powder XRD patterns of MnBi particles and MnBi/Fe-Co (95/5 wt.\% and 90/10 wt.\%) composites.

Figure 8a shows a SEM image of $\mathrm{MnBi} / \mathrm{Fe}-\mathrm{Co}(95 / 5 \mathrm{wt} . \%)$ composites. The surface of $\mathrm{MnBi}$ is decorated with a $20 \mathrm{~nm}$ thick soft shell of Fe-Co, as shown in Fig. 8b. The boundary between the hard and soft phases is marked with an orange dashed line. The soft Fe-Co particles are magnetically attracted to the hard phase MnBi surface by magnetic attraction [9]. The magnetic self-assembly process is further 
explained in Fig. 9. The Fe-Co nanoparticles are well dispersed in hexane because of organic ligand on the surface. The size of $\mathrm{MnBi}$ is reduced by grinding, and the hexane is gradually evaporated. Therefore, the Fe-Co nanoparticles concentrate in the hexane and are magnetically attracted by MnBi particles, forming a core/shell structure. The distribution between Fe-Co and MnBi in MnBi/Fe-Co (95/5 wt.\%) composites in Fig. 8a is investigated by SEM elemental mapping, as shown in Fig. 8c, d, e and f, for the elements of $\mathrm{Bi}, \mathrm{Mn}, \mathrm{Co}$ and $\mathrm{Fe}$, respectively. The mappings show a homogenous distribution between $\mathrm{MnBi}$ and Fe-Co particles, which contributes to the effective exchange coupling between the hard and soft phases in MnBi/Fe-Co (95/5 wt.\%).

To further confirm the core/shell structure of $\mathrm{MnBi} / \mathrm{FeCo}$ composites, the Energy Dispersive X-ray (EDX) spectrum is measured, as shown in Fig. 10. Figs. 10a and 10b show the TEM and Scanning Transmission Electron Microscopy (STEM) images of MnBi/Fe-Co core/shell composites, respectively, and Fig. 10c shows the line scanning of EDX spectrum in Fig. 10b. The red square in Fig. 10b is used to locate a landmark and prevent electron beam drifting. In the EDX spectrum, the signal of Fe and Co is constant along the line, while the signal of $\mathrm{Mn}$ and $\mathrm{Bi}$ is almost zero at both ends of the line, indicating the core/shell structure of $\mathrm{MnBi} / \mathrm{Fe}-\mathrm{Co}$ composites. In addition, more TEM images of $\mathrm{MnBi}$ and $\mathrm{MnBi} / \mathrm{Fe}-\mathrm{Co}$ particles are presented in Fig. 11. Fig. 11a shows a TEM image of a MnBi particle, and Figs. $11 \mathrm{~b}$ and $11 \mathrm{c}$ show the TEM images of $\mathrm{MnBi} / \mathrm{Fe}-\mathrm{Co}$ composites at low and high magnification, respectively. Comparing with Fig. 11a, the particle surface in Figs. 11b and 11c are decorated with a thin layer of nanoparticles, furthering confirming the core/shell structure.

The optimum amount of Fe-Co in exchange coupled $\mathrm{MnBi} / \mathrm{Fe}-\mathrm{Co}$ composites is determined by the size of MnBi. Smaller size MnBi particles provides a larger surface area, so that more Fe-Co nanoparticles can be contacted and exchange coupled with MnBi. However, smaller size of $\mathrm{MnBi}$ particles would result in higher sensitivity to oxidation and the loss of magnetic properties. 


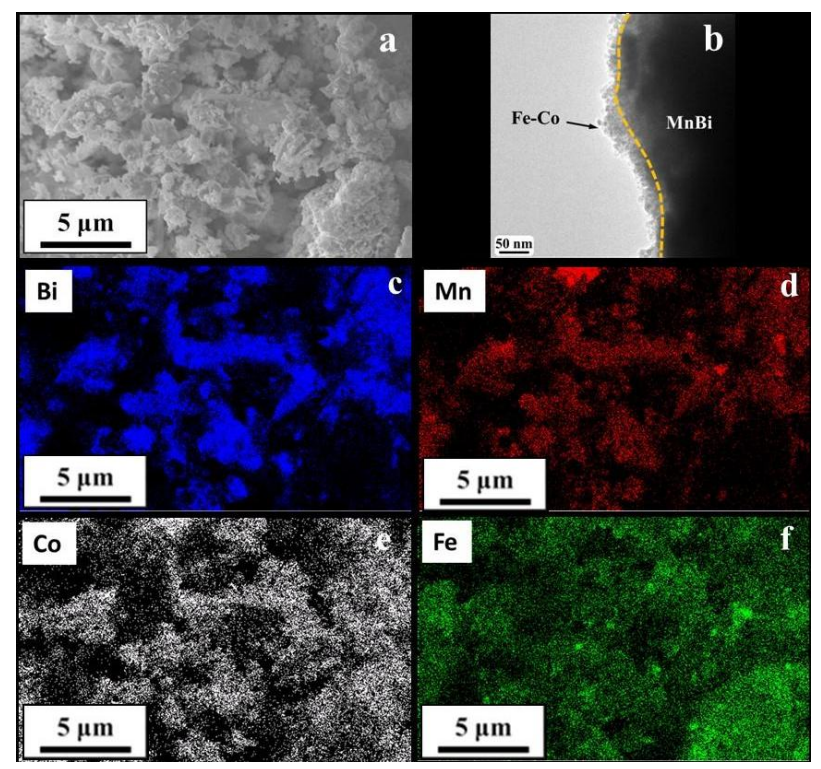

Fig. 8. (a) SEM and (b) TEM images, and SEM elemental mappings of (c) Bi, (d) Mn, (e) Co and (f) Fe for $\mathrm{MnBi} / \mathrm{Fe}-\mathrm{Co}(95 / 5 \mathrm{wt} . \%)$ composites.

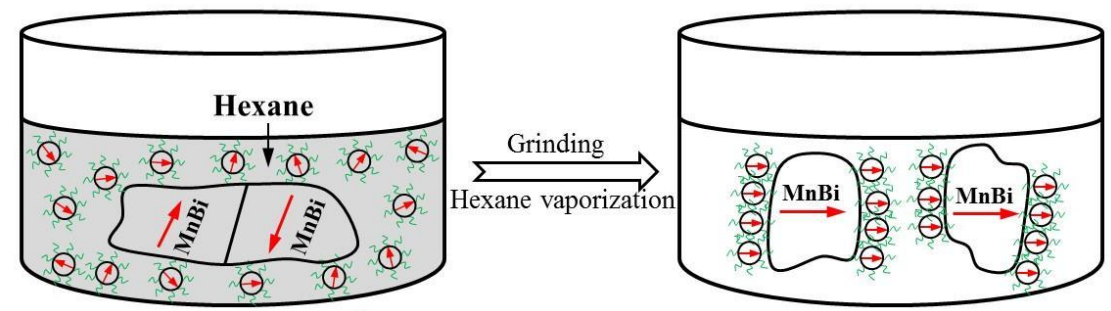

Ligand decorated Fe-Co nanoparticle

Fig. 9. The schematic of magnetic self-assembly process of $\mathrm{MnBi} / \mathrm{Fe}-\mathrm{Co}$ core/shell particles.
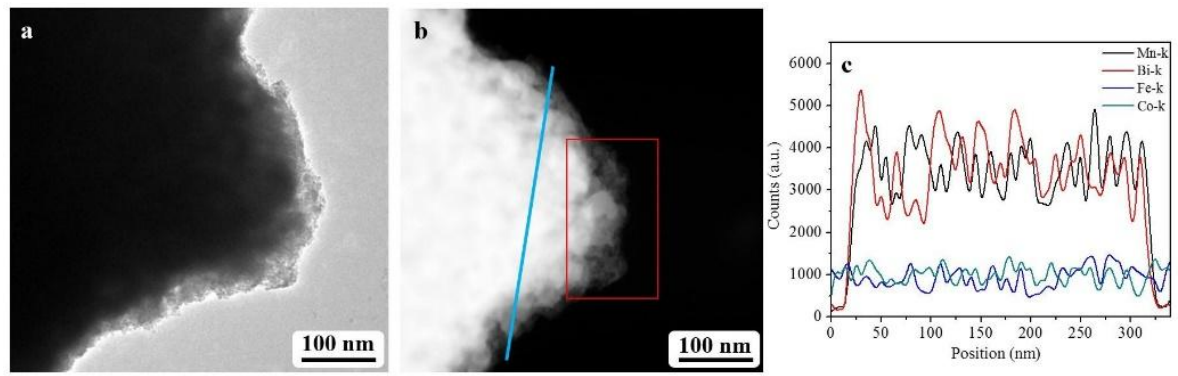

Figure 10. MnBi/Fe-Co particles (95/5 wt.\%): (a) TEM image; (b) STEM image; (c) EDX spectrum of drift corrected line scanning in (b). 

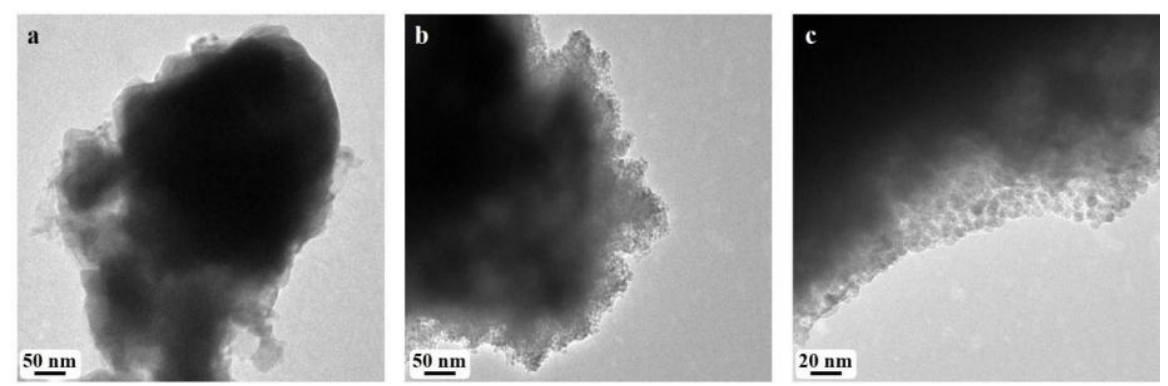

Figure 11. (a) TEM image of MnBi particle; (b) TEM image of MnBi/Fe-Co particle (95/5 wt.\%) under low magnification; (c) TEM image of MnBi/Fe-Co particle (95/5 wt.\%) under high magnification.

\section{Conclusions}

Core/shell structured $\mathrm{MnBi} / \mathrm{Fe}-\mathrm{Co}$ composites were synthesized through magnetic self-assembly, by grinding $\mathrm{MnBi}$ and organic ligand decorated $\mathrm{Fe}-\mathrm{Co}$ nanoparticles in hexane. It was found that effective magnetic exchange coupling occurred in $\mathrm{MnBi} / \mathrm{Fe}-\mathrm{Co}(95 / 5 \mathrm{wt} . \%)$ composites, which was confirmed by the single-phase-like hysteresis loop, increased remanent magnetization and dominant positive peak in $\Delta M$ curves.

\section{Acknowledgement}

This work was supported in part by the U.S. Department of Energy ARPA-E REACT Program under Award Number DE-AR0000189.

\section{References:}

[1] C.B. Rong, N. Poudyal, J.P. Liu, Phys. Lett. A. 374 (2010) 3967-3970.

[2] J. Wecker, M. Katter, L. Schultz, J. Appl. Phys. 69 (1991) 6058-6060.

[3] M.F. de Campos, F.J.G. Landgraf, N.H. Saito, S.A. Romero, A.C. Neiva, F.P. Missell, E. de Morais, S. Gama, E.V. Obrucheva, B.V. Jalnin, J. Appl. Phys. 84 (1998) 368-373.

[4] S. Sugimoto, J. Phys. D: Appl. Phys. 44 (2011) 064001.

[5] X. Xu, J. Park, Y. K. Hong, A. M. Lane, Mater. Lett. 144 (2015) 119-122

[6] T.J. Nummy, S.P. Bennett, T. Cardinal, D. Heiman, Appl. Phys. Lett. 99 (2011) 3671329.

[7] X. Xu, J. Park, Y. K. Hong, A. M. Lane, J. Solid. State. Chem. 222 (2015) 84-89.

[8] J. Cui, J.P. Choi, G. Li, E. Polikarpov, J. Darsell, N. Overman, M. Olszta, D. Schreiber, M. Bowden, T. Droubay, M.J. Kramer, N.A. Zarkevich, L.L. Wang, D.D. Johnson, M. Marinescu, I. Takeuchi, Q.Z. Huang, H. Wu, H. Reeve, N.V. Vuong, J.P. Liu, J. Phys. Condens. Mat. 26 (2014) 064212.

[9] X. Xu, J. Park, Y. K. Hong, A. M. Lane, Mater. Chem. Phys. 152 (2015) 9-12

[10] J. Park, Y.K. Hong, J.J. Lee, W.C. Lee, S.G. Kim, C.J. Choi, Metals. 4 (2014) 455-464. 
[11] P. Kharel, P. Thapa, P. Lukashev, R.F. Sabirianov, E.Y. Tsymbal, D.J. Sellmyer, B. Nadgorny, Phys. Rev. B. 83 (2011).

[12] X. Guo, X. Chen, Z. Altounian, J.O. Stromolsen, Phys. Rev. B. 46 (1992) 14578-14582.

[13] B.W. Roberts, Phys. Rev. 104 (1956) 607-616.

[14] Y.L. Ma, X.B. Liu, K. Gandha, N.V. Vuong, Y.B. Yang, J.B. Yang, N. Poudyal, J. Cui, J.P. Liu, J. Appl. Phys. 115 (2014) 4868078.

[15] Y.K. Hong, J. Park, O.N. Mryasov, S.G. Kim, S. Kim, J. Lee, G.S. Abo, C.J. Choi, J. Lee, AIP. Adv. 3 (2013) 4809564.

[16] R. Skomski, J.M.D. Coey, Phys. Rev. B. 48 (1993) 15812-15816.

[17] R. Skomski, J.M.D. Coey, IEEE. T. Magn. 30 (1994) 607-609.

[18] R. Skomski, J. Appl. Phys. 76 (1994) 7059-7064.

[19] J. Shen, H.Z. Cui, X.P. Huang, M.G. Gong, W. Qin, A. Kirkeminde, J. Cui, S.Q. Ren, RSC. Adv. 5 (2015) 5567-5570.

[20] J.F. Bondi, K.D. Oyler, X.L. Ke, P. Schiffer, R.E. Schaak, J. Am. Chem. Soc. 131 (2009) 9144-9145.

[21] B.D. Cullity, C.D. Graham, Introduction to Magnetic Materials, Wiley2009.

[22] K. Kang, L.H. Lewis, A.R. Moodenbaugh, Appl. Phys. Lett. 87 (2005).

[23] Y. Wang, J.F. Wong, X.W. Teng, X.Z. Lin, H. Yang, Nano. Lett. 3 (2003) 1555-1559.

[24] A.L. Patterson, Phys. Rev. 56 (1939) 978-982.

[25] E.F. Kneller, R. Hawig, IEEE. T. Magn. 27 (1991) 3588-3600.

[26] Y. Hou, S. Sun, C. Rong, J.P. Liu, Appl. Phys. Lett. 91 (2007) 153117. 


\section{Magnetic Self-Assembly for the Synthesis of Magnetic Exchange Coupled MnBi/Fe-Co Composites}

Xia Xu ${ }^{a}$, Yang-Ki Hong ${ }^{\mathrm{b} 1}$, Jihoon Park $^{\mathrm{b}}$, Woncheol Lee ${ }^{\mathrm{b}}$, Alan M. Lane ${ }^{\mathrm{a}}$, and Jun Cui ${ }^{\mathrm{c}}$

${ }^{a}$ Department of Chemical and Biological Engineering and MINT Center, The University of Alabama, Tuscaloosa, AL 35487 USA

${ }^{\mathrm{b}}$ Department of Electrical and Computer Engineering and MINT Center, The University of Alabama, Tuscaloosa, AL 35487 USA

${ }^{c}$ Energy and Environment Directorate, Pacific Northwestern National Laboratory

902 Battelle Boulevard, Richland, WA 99352

\section{Graphic Abstract}

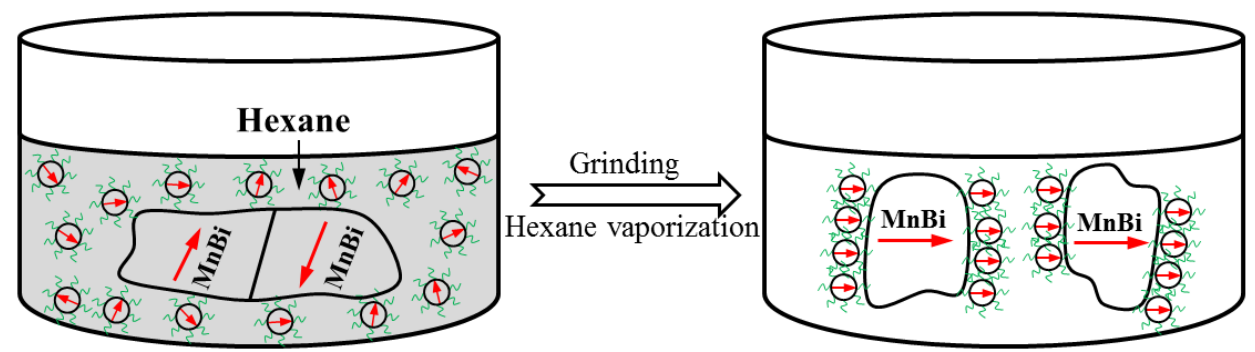

$\Theta_{2}$ Ligand decorated Fe-Co nanoparticle

${ }^{1}$ Corresponding author: phone: 205-348-7268; fax: 205-348-6959; email: ykhong@eng.ua.edu 
Magnetic Self-Assembly for the Synthesis of Magnetic Exchange Coupled MnBi/Fe-Co Composites

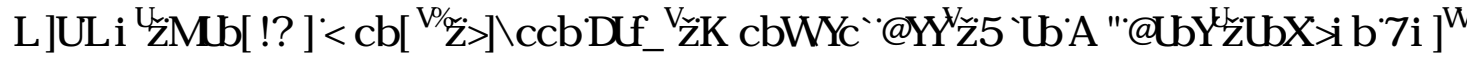

D HSDUN HQNRI \&KP IFDODG\%RRRJ IFDO Q LQHULI DQG0 , 17 \&HQMU 7 KH8 QIYHWW RI \$ DEDP D 7 XVFDRRVD \$ 86 \$

E. HSDUI HQWRI ( OFWNFDODQG\&RP SXWU( Q LQHUQ DQG0 ,1 7 \&HQMU 7 KH8 QYYHWW RI \$ DEDP D 7 XVFDRRVD \$ $\quad 86 \$$

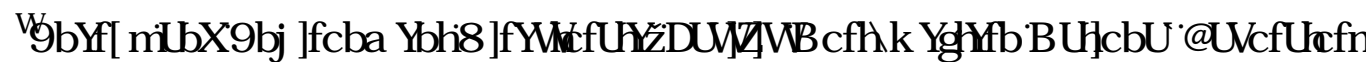

\%DNOOH\%XOMDG 5 IFKOOOG : \$

Graphic Abstract Legend

\%RUK0 Q/LDOG) H\&R SDWFONZHHGLSHUGLQKH DQHIRUJ UQCQ \%HDXVRI UKHROF DFG

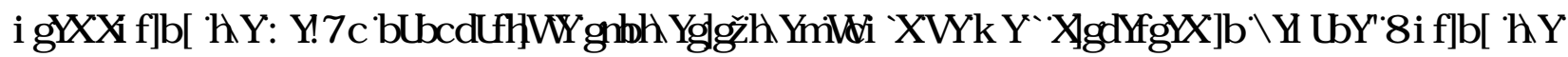

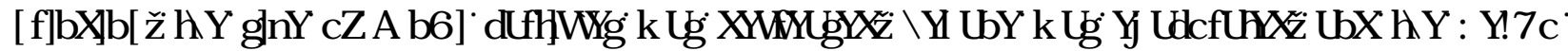

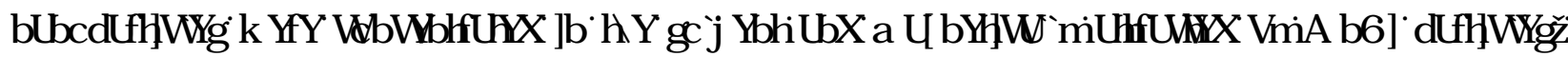
IRUP IQI DFRLHWHOWXFXIH 